\title{
Developing Hybrid Car to Car Communication System Based on MIMO Visible Light and Radio Frequency
}

Nermeen Maged Okasha ( $\square$ nermena2012@gmail.com )

Ain Shams University Faculty of Engineering https://orcid.org/0000-0002-5418-7304

AbdelHalem A. Zekry

Ain Shams University Faculty of Engineering

Fatma A. Newagy

Ain Shams University Faculty of Engineering

\section{Research Article}

Keywords: Fog Effect, Rain Effect, Hybrid RF, Hybrid VLC, MIMO VLC, Car to Car.

Posted Date: January 10th, 2022

DOI: https://doi.org/10.21203/rs.3.rs-1132769/v1

License: (c) This work is licensed under a Creative Commons Attribution 4.0 International License.

Read Full License 


\title{
Developing hybrid car to car communication system based on MIMO
}

\section{visible light and radio frequency}

\author{
Nermeen M. Okasha ${ }^{a, b, *}$, Abdel Halem A. Zekry ${ }^{a}$, Fatma A. Newagy $^{a}$ \\ ${ }^{a}$ Electronics and Communications Engineering Department, Faculty of Engineering, Ain shams University, Cairo, Egypt. \\ ${ }^{b}$ Electronics and Communications Engineering Department, Modern Academy for Engineering and Technology, Cairo, Egypt. \\ *Correspondence e-mail: Nermena2012@gmail.com
}

Abstract - This paper combines the advantages of both VLC communication and RF communication for car-to-car applications to achieve a higher data rate, more range coverage, smaller delay, and smaller BER. In the proposed scheme, the VLC maximum communication distance is chosen as $100 \mathrm{~m}$ per-hop approximately, which can be extended through multi-hop up to the timeout window of 5 hops. In contrast, the RF maximum communication distance is chosen as $200 \mathrm{~m}$ per-hop approximately, which is reasonable at the frequency band of $70 \mathrm{GHz}$ to $90 \mathrm{GHz}$ due to the high attenuation at this frequency band. A MATLAB simulation for a car-to-car framework is built to demonstrate and compare the BER, throughput, and delay outcomes at a hybrid VLC and RF communication. Our results show that VLC can achieve up to four times of the RF throughput while maintaining low BER of $10^{-6}$ and small delay of $10^{-4}$ with respect to $\mathrm{RF}$ communication only.

Keywords - Fog Effect, Rain Effect, Hybrid RF, Hybrid VLC, MIMO VLC, Car to Car.

\section{INTRODUCTION}

RF communication is not always the best communication solution, so many researchers are looking for alternatives. One of the most popular alternatives is visible light communication (VLC), because of the modern optical communication breakthrough. We will show the pros and cons of both RF and VLC car-to-car communication that motivates us to build a hybrid system to harvest the benefits from both of them and overcome the limitation of each of them. 
The Popularity of RF communication leads to overbooking in all its bands. New applications are trying to coexist with the current ones. However, there are no reserved bands for these applications. Then, one of the possible solutions is to remove a working application from its band and replace it with a new application in the same band (e.g., replacing the $3 \mathrm{G}$ mobile network with $5 \mathrm{G}$ ) [1]. That means all mobiles that support only $3 \mathrm{G}$ will stop working and their owners must buy new $5 \mathrm{G}$ phones to get the same service again [2]. Moreover, the same problem will affect all other wireless devices (i.e., tv, radio, Wi-Fi, etc.).

On the other hand, the possible solution for RF spectrum scarcity is frequency reuse by both legacy and new applications through introducing possible separators (e.g., time, code, power, location, etc.) $[3,4]$. However, it is only a temporary solution and limits new applications' horizons while introducing more complexity to the system. For example, to decrease the interference in the system we must decrease the power of each tower which leads to dead zones and calls drops increase [5]. In addition, to refill these zones more towers are added which increases the overall system cost and so on. This cycle appears in every aspect of the RF communication, enhancing any parameter affecting all other parameters with a global limitation to the achieved data rate [6].

The appearance of smart new applications (e.g., Calls and video with hologram, auto drive, etc.), which need a higher data rate than the RF can cover, open the way to other communication techniques such as visible light communication [7]. The VLC communication is popular of its higher data rate which can cover modern applications' needs.

Also, the spread of industrial light sources technology and devices (e.g., LED, light detector) enables cost-effective VLC communications [8]. VLC has an easy and small constellation to set up a communication link (i.e., LED, camera) at home where we can use the house light sources to communicate with our mobile phones' cameras. At the street, we can use the street lights with our mobile cameras or the car headlight with another car parking camera. In a short distance, the VLC communications show a smaller bit error rate (BER) compared to RF at the same distance and power 
level because it has a smaller noise level. However, faraway links cannot be achieved through VLC and need another means of communication $[9,10]$.

Consequently, this paper combines the advantages of both VLC communication and RF communication for car-to-car applications. By merging VLC with RF, the hybrid communication system achieves higher range coverage and smaller BER.

The authors in [11] proposed selective relaying based on the transmitted data type. They categorized the data types into two major types. On the one hand, the important control short packets that need to reach the receiver even if the delay time is longer than expected. On the other hand, the large amount of downloading and streaming packets that require large bandwidth, small delay, and able to accept packet drop percentage. In their algorithm, the authors use Coalitional Game (CG) and Analytic Hierarchy Process (AHP) to separate the packets' flow for each type based on the car-to-car communication path properties. However, that algorithm recomputes each path property when a car enters or exits the neighboring zone which drains the system resources (e.g., batteries, processing). Moreover, the proposed system uses only RF communication which has a limited bandwidth and suffers from a high level of interference.

While the authors in [12], proposed an air channel for the VLC communication with variable levels of foggy weather. The fog level is expressed through the meteorological visibility which varies from $10 \mathrm{~m}$ up to $10 \mathrm{~km}$. However, what affects the VLC communication is meteorological visibility between $10 \mathrm{~m}$ and $500 \mathrm{~m}$. The authors proposed that the transmitter is LED and the receiver is a camera. For a constant distance between two cars and variable fog meteorological visibility, the BER is small for meteorological visibility up to $20 \mathrm{~m}$ at different LED light modulations. However, for meteorological visibility of $10 \mathrm{~m}$ or less and at the same LED light modulations the BER shows a dramatic increase that leads to consider the communication link as down. In contrast, no solution is proposed such as using the RF communication under this condition. Moreover, no study is presented at the multi-hop VLC communication under the fog weather condition. 
The authors in [13] proposed a practical VLC communication system where the proposed system uses 4G telecom infrastructure as a backup. The VLC communication uses a single laser source as a transmitter and a light detector as a receiver. However, the throughput of the presented system is only $50 \mathrm{kbit} / \mathrm{s}$ at $10^{-7} \mathrm{BER}$ and within small communication distances less than $50 \mathrm{~m}$. The authors did not present detailed results to the VLC or RF communication in a car to car. Moreover, no MIMO is proposed to increase the VLC data rate.

The authors in [14] built a full VLC channel model from the car headlight as the source to the camera as the receiver that includes the distance between cars, the difference in azimuth, and elevation angles due to different road lanes and road irregular surface respectively. The suspension system of each car that moves on irregular roads tries to compensate for the generated vibrations. However, these vibrations cause differentiation in the elevation angles that degrade the received signal by at most $4 \mathrm{~dB}$.

On the other hand, different road lanes cause a change in the azimuth angles that degrades the received signal by at most $3 \mathrm{~dB}$ within the light beam field of view (i.e., half-power beamwidth) and are considered as blocked otherwise. Consequently, the change in azimuth and elevation angles lead the VLC communication to degrade in longer distances which is the main required parameter. However, the authors did not propose a solution to lower VLC car-to-car communication distance such as multi-hop or using RF as a backup means of communication.

The authors in [15] made a comparison between the communication through VLC and the communication through infrared. The authors built a practical model using real cars and used the engine control unit (ECU) of the car as a processing resource, the headlights as the transmitter, and a photodetector as the receiver. However, the presented results are somehow below the expectation because of the use of a photodetector as the receiver and not a camera. They study the weather effect on both the VLC and infrared and find out that VLC is less effective than infrared at foggy conditions. 
In contrast, VLC is better than infrared in all other weather conditions because the background ambient temperature of the cars works as interference in infrared communication that lowers its SNR.

In this paper, we aim to benefit from the modern technology of automotive manufacturing. Firstly, we use the car's LED light and parking cameras to form VLC communication links between cars to transmit road data. Moreover, we benefit from VLC's high data rate and small error rate for short coverage. Then, we extend the VLC communication coverage in the distance out of VLC single-hop range using the multi-hop technique by making other cars within the road work as repeaters between the source and destination while maintaining the high data rate and small error rate. Finally, when VLC reaches its time out of hopes, more coverage is realized by using RF communication which is known for its larger coverage even if its data rate is smaller. Therefore, by merging VLC with RF, the hybrid communication system assumes high range coverage and small BER. Our study is devoted to determining the performance parameter of the hybrid system including bit error rate BER, throughput and delay under clear weather, heavy fog, and heavy rain conditions.

The rest of this paper is organized as follows: Section 2 describes the methods/experimental of the study. Section 3 describes the system model. Section 4 presents the proposed VLC multi-hop communication system. Section 5 depicts the simulation results and their analysis then section 6 concludes the paper.

\section{METHODS/EXPERIMENTAL}

\subsection{The Design of The Study}

The Research design is a MATLAB framework of 80 cars within six lanes road with three lanes in one direction and the other three lanes in the opposite direction. The communications among the cars will be modeled, simulated, and analyzed through some metrics. The type of research is semiexperimental while its sub-type is a research problem whether the hybrid VLC/RF communication has improved metrics compared to RF only or not. 


\subsection{The Setting}

After importing the recorded movement of the cars on the selected road into the MATLAB simulation file, we select one of the cars at random as the transmitter and another car as the receiver. The transmitter car generates the data and starts forming a connection with the receiver either through VLC or RF as will be illustrated. Once the connection is established, the MATLAB program measures the needed metrics. Finally, we analyze the output charts to determine the effectiveness of the study.

\subsection{The Type of Participants or Materials Involved}

We have recorded the movement of 80 cars in a section of the ring road in Cairo, Egypt at dawn. The recorded data contains locations at different time stamps. From that data, we can compute each car's speed.

\subsection{A Clear Description of All Interventions and Comparisons}

In results section 5 .

\subsection{The Type of Analysis Used}

We have chosen the inferential analysis, as it uses samples from the world to test the authors' theory.

\section{SYSTEM MODEL}

As shown in fig. 1, our car-to-car communication system comprises a framework of 80 cars within six lanes road with three lanes in one direction and the other three lanes in the opposite direction. Whose communications will be modeled and simulated using MATLAB. In fig. 1 green arrows represent single-hop VLC, red arrows represent multi-hop VLC, yellow arrows represent multi-hop VLC to overcome the field of view problem while yellow lightning represents single-hop RF. 


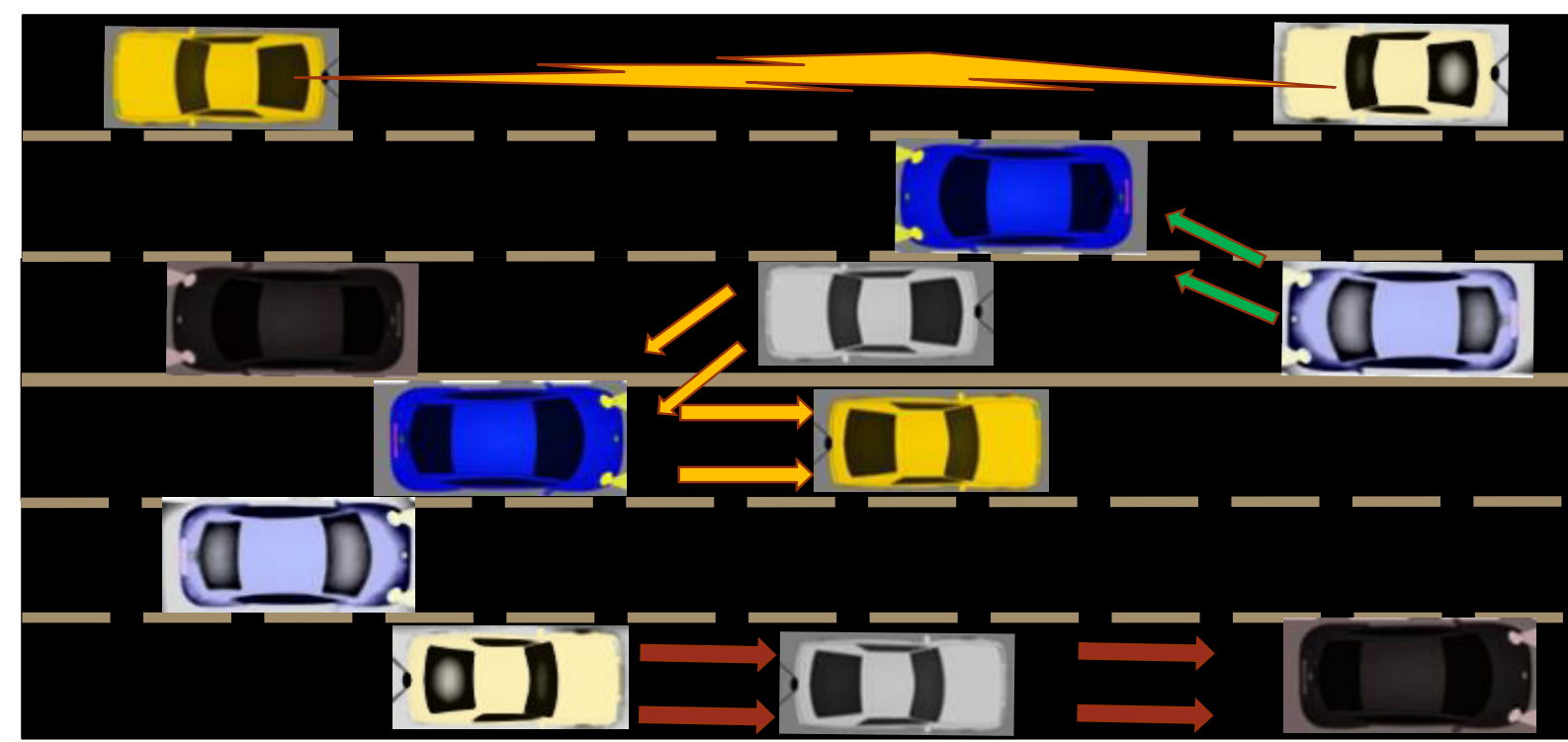

Figure 1 The proposed framework of 80 cars communicates through VLC/RF within 6 lanes road (3 lanes in each direction)

The selected road distance is $25 \mathrm{~km}$. Each car has a variable speed from $60 \mathrm{~km}$ to $100 \mathrm{~km}$. To reduce the randomness in the results, a Mount Carlo of 120 simulations is performed. Also, these different simulations, i.e., different senders to different destinations, add robustness to the results. In each simulation, a transmission data set of $100 \mathrm{kbit} /$ packet is established from a sender to a destination through the available communication type as single-hop - multi-hops - and RF to test the algorithm operation. The VLC maximum communication distance is chosen as $100 \mathrm{~m}$ approximately which can be extended through multi-hop up to the timeout window of 5 hops.

In contrast, the RF maximum communication distance is chosen as $200 \mathrm{~m}$ approximately which is reasonable at the frequency band of $70 \mathrm{GHz}$ to $90 \mathrm{GHz}$ due to the high attenuation at this frequency band. The number of LEDs in each car headlight is 9 in a 3 by 3 grid each of them sends transmission data independently while the receiver camera receives all of them at once through the captured frame processing. The camera captures up to 2000 frames in each second. Table 1 summarizes our simulation parameters. 
Table 1 MATLAB model simulation parameters

\begin{tabular}{|lc|}
\hline \multicolumn{1}{|c|}{ Parameter } & Value \\
\hline Number of cars & 80 cars \\
\hline Simulation Lanes & 6 lanes \\
\hline Road Distance & $25 \mathrm{~km}$ \\
\hline Car Speed & $60 \sim 100 \mathrm{~km} / \mathrm{hour}$ \\
\hline Length of Data & $100,000 \mathrm{bits}$ \\
\hline MIMO & 9 input / camera output \\
\hline Camera & $2000 \mathrm{FPS}$ \\
\hline Timeout Window & $5 \mathrm{hops}$ \\
\hline Mount Carlo & 120 simulations \\
\hline VLC Distance & $\sim 100 \mathrm{~m}$ \\
\hline RF Distance & $\sim 200 \mathrm{~m}$ \\
\hline RF frequency & $70 \sim 90 \mathrm{GHz}$ \\
\hline
\end{tabular}

\subsection{Communication Through Visible Light:}

In the communication through visible light, the transmitter is a photonic source (i.e., laser or LED), the propagation media is air with all its weather conditions (e.g., sunny, foggy, rainy, or night), and the receiver is a photonic absorber generally a photodetector or especially a camera. In car-to-car communication, modern cars have built-in LED lights and sensor cameras. Then, this paper proposes using the LED light as a VLC transmitter and the camera as the receiver.

The characteristics of the car-to-car VLC system LED source is as follows. The LED white light occupies all the visible light emission bandwidth from $430 \mathrm{THz}$ to $740 \mathrm{THz}$. Each car headlight is divided into 9 independent LED lights in a 3 by 3 grid to form multiple input algorithms each of them transmits independently using ON OFF key (OOK) modulation.

The communication channel of VLC is normally modeled as additive white Gaussian noise (AWGN) with a specific attenuation coefficient depending on the communication distance. The channel is affected by different weather conditions such as fog, sand storms, or rain. In addition, external interference such as street lights, commercial boards, and sunlight affect the VLC communication. 
The receiver camera captures shoots from 30 frames per second (FPS) up to 2000 FPS. Higher FPS increases the data rate. Each shot captures all the transmitter car headlight LEDs. Then, the receiver camera receives all of the 9 LEDs at once through each captured frame. The captured camera shot is processed to identify the LED grid and an illumination threshold per LED is set to distinguish the received data either ones or zeros. The multiple inputs LED grid and the camera output shots form a MIMO system to increase the VLC data rate.

\subsection{Channel Characteristics}

Beer's law is used to determine the fog channel coefficient $H_{F O G}$ [22]. Beer's law describes the absorption and scattering of light in the air as a medium at different visibility levels. As this paper is concerned about the short-range car to car communication then Beer's law can be simplified for meteorological visibility $(V)$ less than $0.5 \mathrm{~km}$ as:

$$
H_{F o g}=e^{-\left(\frac{3.91}{V}\right)}
$$

Where $V$ is the meteorological visibility in kilometers. In addition, line of sight channel coefficient $H_{L O S}$ for free space light communication follow the general free space loss formula:

$$
H_{L O S}=\frac{A \cos (\theta) \cos (\varphi)}{2 \pi D^{2}}
$$

Where $A$ is the amplitude of light in illuminance, $\theta$ and $\varphi$ are the transmitter and receiver angles from the communication line of sight centerline, and $D$ is the distance from the transmitter to the receiver. Moreover, to compute the rain channel coefficient $H_{\text {Rain }}$ that depends on temperature, rain size, and frequency, we adopt the Marshal and Palmer distribution of raindrop size [5]. In Marshal and Palmer's formula, the wireless optical attenuation is related to the rain rate $(R R)$ in millimeter per hour, and the parameters of power-law $A=0.365$ and $B=0.63$. Then, $\gamma$ in $\mathrm{dB} / \mathrm{km}$ equals to:

$$
\gamma=A \times R R^{B}
$$




$$
H_{\text {Rain }}=\frac{4 \lambda}{\pi \times h c} \times 10^{-\gamma L / 10}
$$

Where $\lambda$ is the wavelength, $h$ is the Planck constant, and $c$ is the speed of light in free space. Note that to make the Marshal and Palmer assumption independent of polarization, we assume a spherical shape raindrop.

Finally, the received light signal captured by the camera is equal to the transmitted OOK modulated light multiplied by all the aforementioned channel coefficients and adding the additive white Gaussian noise as shown in Figure 2, then:

$$
Y_{R x}=H_{L O S} H_{R a i n} H_{F o g} X_{T x}+N_{A W G N}
$$

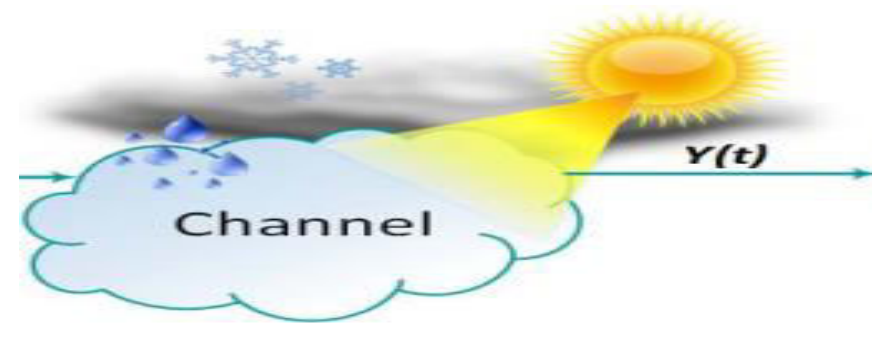

Figure 2 the VLC Channel Characteristics at different weather conditions such as fog, rain, or direct sunlight.

\subsection{RF Communication:}

In RF communication, the transmitter is an electromagnetic emitter with carrier frequency from 70 $\mathrm{GHz}$ to $90 \mathrm{GHz}$ and an omnidirectional antenna. In the $70 \mathrm{GHz}$ to $90 \mathrm{GHz}$ frequency band, the assigned bandwidth is $2 \mathrm{GHz}$ approximately (i.e., the highest compared to lower bands). However, the attenuation in this band is also higher than other bands which decreases the communication distance that limits the applications in such band. Then, this band has limited applications and one of them is car-to-car communication. The RF receiver is an electromagnetic detector with an Omnidirectional antenna in both line-of-sight and non-line-of-sight communication. The RF communication channel is less affected by the VLC channel attenuation conditions in the air (e.g., fog, walls, or sandstorm). However, both VLC and RF channels are affected by attenuation in metals or concretes. In this paper, 
we assume 1024 QAM modulation and the RF critical transmission range is $200 \mathrm{~m}$ because of the high attenuation level. In addition, increasing the QAM beyond 1024 dramatically decreases the SNR and leads to decreased distance. All cars' transmission through RF is considered interference to each other. Then, we need to minimize the number of cars using RF communication and make them more dependent on VLC communication.

\section{THE PROPOSED HYBRID VLC AND RF SCHEME}

As shown in the flow chart in Figure 3, the proposed scheme tries to communicate through VLC single-hop then increases the number of hops if it fails to close the connection. The proposed scheme aims to achieve high car-to-car throughput and overcome the coverage limitations. However, in some weather conditions, the VLC communication cannot succeed. Then, if the maximum number of hops is reached, the scheme switches the communication type to RF.



Figure 3 the proposed scheme flowchart.

\subsection{Transmission through VLC:}

If the receiver is within the VLC line-of-sight of the transmission source, academia calls it a singlehop transmission. The VLC line-of-sight has two limitations, distance and the angle of view. Then, if 
the receiver is out of the VLC communication distance even if it is within the angle of view or out of the angle of view even if it is within the communication distance, the receiver fails to perform singlehop transmission. In addition, any obstacle that blocks the line-of-sight between the transmitter and the receiver will fail the single-hop transmission. The proposed scheme always tries to communicate through single-hop VLC first as shown in the flowchart.

In contrast, if the transmitter did not find the receiver within its single-hop range, the transmitter records all the reachable cars as relays. The relays search for the receiver within their VLC line-of-sight range. If the receiver is found, then, the nearest relay reports back to the transmitter to start the communication session. Otherwise, if all relays did not find the receiver, then, the scheme starts another round of relays to find the receiver. Figure 4 shows the multi-hop concept. In Figure 4, the transmitter source (i.e., white car) communicates through the repeater (i.e., blue car) to reach the receiver (i.e., yellow car). Therefore, multi-hop communication forms a chain of repeaters between the source and destination. An important remark is that if the scheme is left to increase the relays pool for infinity, the receiver may not be found at all and the system fails. Consequently, the proposed scheme introduces the hop counting feature with a maximum number of hops as $W_{\text {max }}$. When the maximum number of hops is reached, the scheme switches to the RF communication. Moreover, if a repeater receives the same message more than once, it discards all the replicas to prevent loops within the repeaters chain.

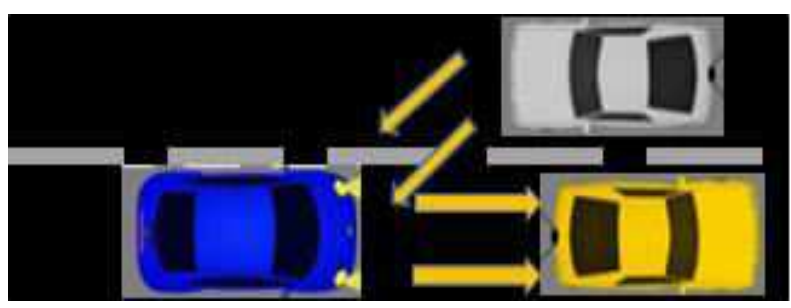

Figure 4 multi-hop car-to-car communication.

\subsection{Transmission through $R F$}

After reaching the maximum number of hops in VLC communication, the scheme shifts to RF communication at distances greater than $100 \mathrm{~m}$ (i.e., after the VLC hop maximum communication 
distance) up to $200 \mathrm{~m}$. In RF, the omnidirectional and non-line of sight features make it easier to reach the destination. However, we consider no communication at distances greater than $200 \mathrm{~m}$ to ensure the minimized interference in $\mathrm{RF}$ and to maximize the frequency reuse in car-to-car RF communication.

In other words, the RF communications from other cars act as interference to each other. Consequently, a higher interference level decreases the RF data rate and may drop the communication link entirely. Therefore, having a limited communication distance and minimizing the dependence on the RF channel keeps the interference level below the assigned threshold for those cars in real need for RF communication type within the scenario and also preserves an acceptable data rate.

\section{RESULTS AND DisCUSSION}

A MATLAB simulation for the aforementioned car-to-car framework is built to demonstrate and compare the outcomes at VLC and RF communication. In each simulation of the 120 Mount Carlo simulations, a transmitter car and a receiver car enter the $25 \mathrm{~km}$ road and start trying to communicate with each other through VLC or RF and use the other cars in the road as repeaters. With the varying speed, the distance between the transmitter and receiver cars may get smaller or larger.

Consequently, the communication type changes through the simulation period. Each packet of 100 kbit is received with some errors because of the noise and weather conditions as in equation (5). The received packet is compared to its original transmitted packet to determine the errors and then calculate the BER. The number of received packets during a simulation determines the system throughput in this simulation. On the other hand, the delay is computed as the needed time for the communication beam to travel the distance between the cars plus the needed processing time in each repeater to receive the data and retransmit it.

The BER versus distance is studied in Figure 5. In VLC communication, increasing the distance leads to higher BER until approximately $100 \mathrm{~m}$ (i.e., the maximum allowed distance for VLC communication) in each hop. In VLC first hop, a small BER with a max of $6.3 \times 10^{-6}$ is noticed. In 
the second hop, the error increased due to the repeater amplify and forward scheme errors with a max of $8.1 \times 10^{-6}$. At higher hops max errors of $1.3 \times 10^{-5}, 1.85 \times 10^{-5}$, and $2.55 \times 10^{-5}$ are measured for third, fourth, and fifth hop respectively.

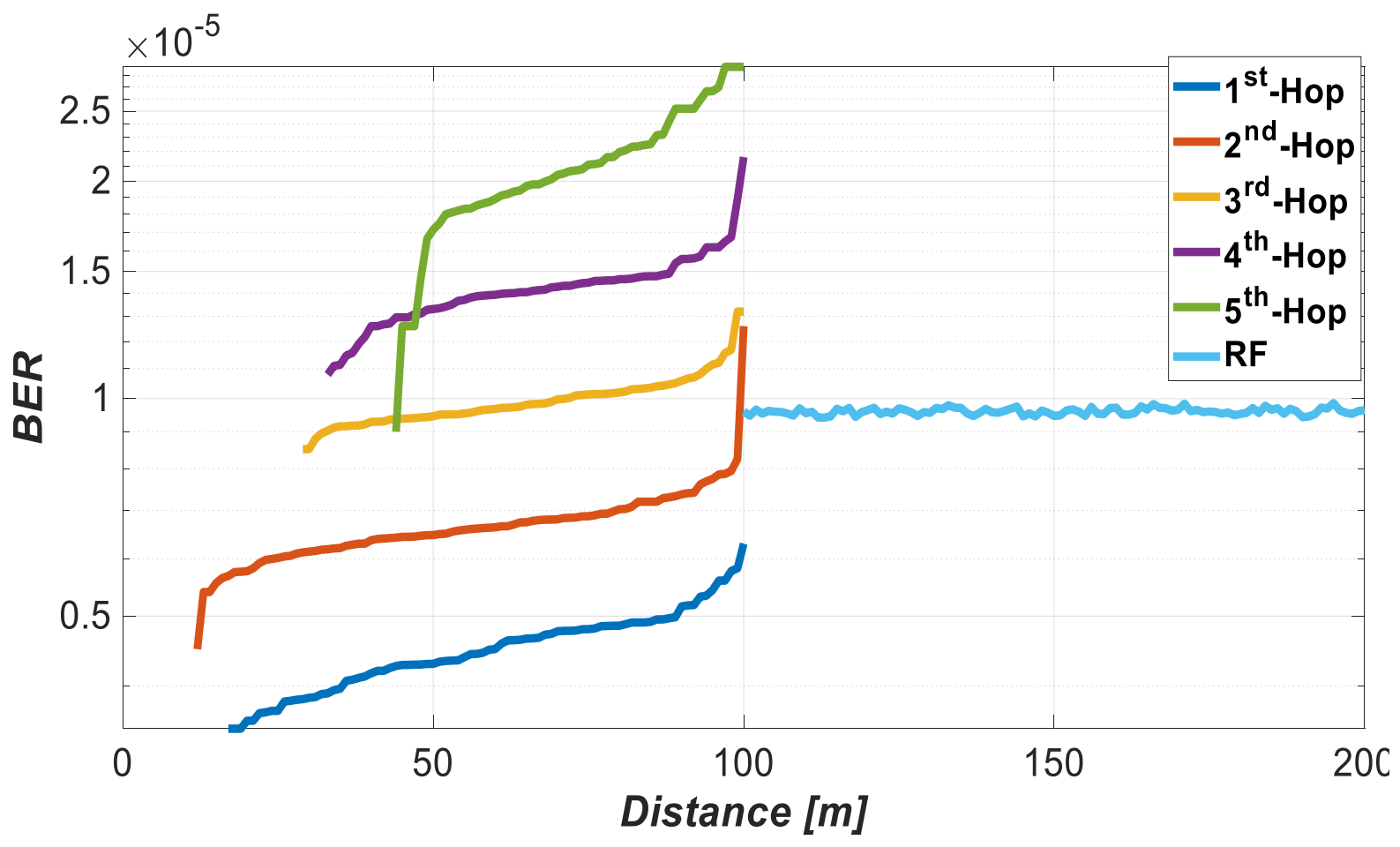

Figure 5 the BER versus distance

On the other hand, in RF communication, we measure the BER at distances from $100 \mathrm{~m}$ (i.e., after the VLC hop maximum communication distance) up to $200 \mathrm{~m}$. we consider no communication at distances greater than $200 \mathrm{~m}$ to ensure the minimized interference in RF (omnidirectional radiation pattern) and to maximize the frequency reuse in car-to-car RF communication. Distance variation has a small effect on RF communication BER with an average of $9.7 \times 10^{-6}$ while as distance reaches $200 \mathrm{~m}$ a very small increase in BER is noticed. Normally in RF communication, the communication distance is much larger than the allowed for car-to-car communication and then the BER shows small variations under that range of distances.

The BER at different weather conditions (i.e., clear weather, heavy fog, and heavy rain) in all communication scenarios are studied and the results are depicted in Figure 6. In general, the VLC and RF communication at clear weather conditions have the lowest BER compared to other weather 
conditions. While, the VLC and RF communication at foggy weather has the highest BER at VLC with low noticeable effect on RF because the fog even if it minimizes the visibility in the visible light band, does not block the other electromagnetic radiations such as RF communication.

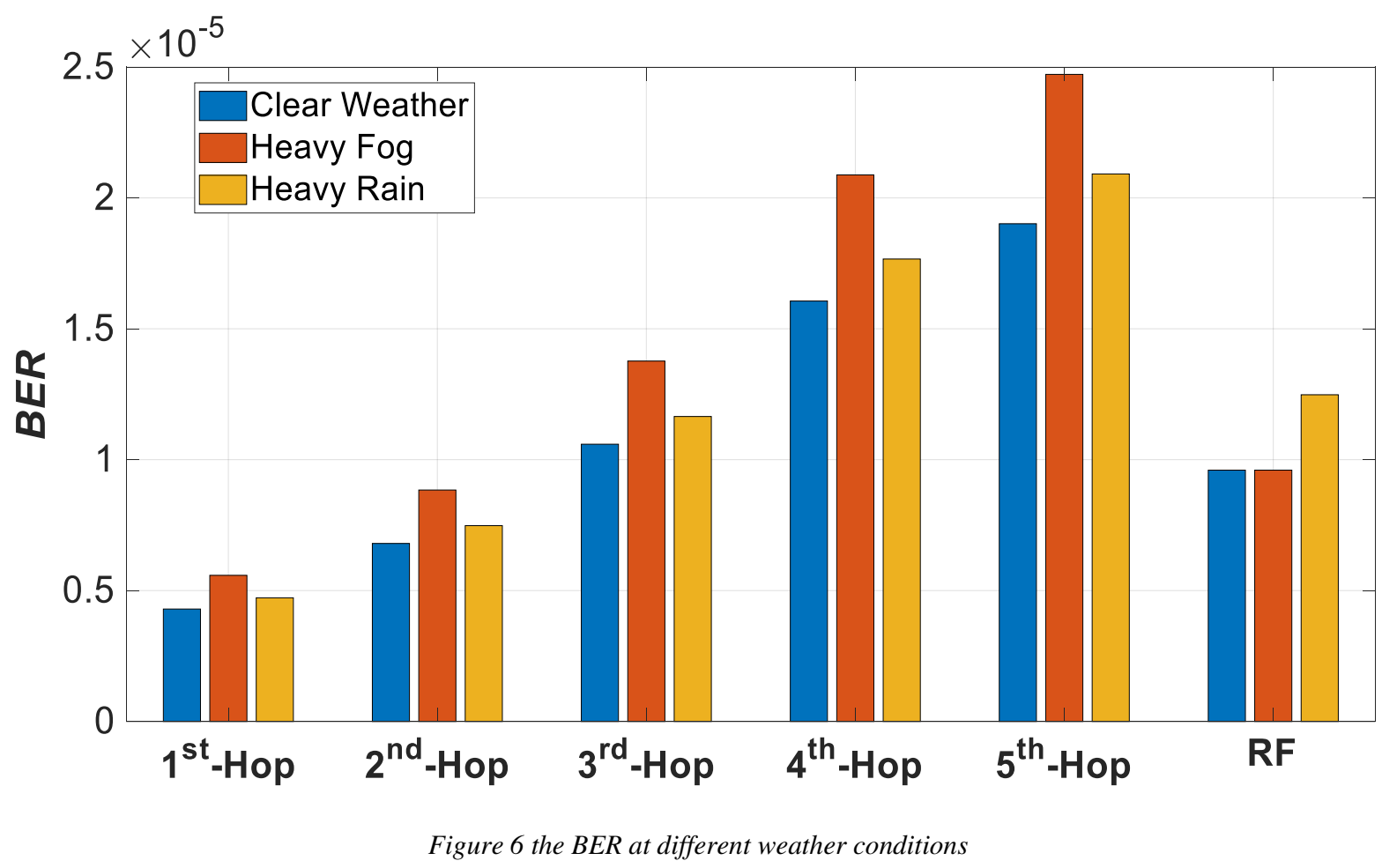

In contrast, the rainy weather has a moderate effect on VLC communication and an increased BER effect on RF communication because the water drops scatter the visible light band beam and absorb (attenuate) the RF electromagnetic beam. On the other hand, the BER for a smaller number of VLC hops (i.e., first hop of $4.6 \times 10^{-6}, 5.2 \times 10^{-6}$, and $4.9 \times 10^{-6}$ and second hop of $6.9 \times 10^{-6}$, $8.1 \times 10^{-6}$, and $7.5 \times 10^{-6}$ ) is lower compared to RF communication BER of $9.6 \times 10^{-6}, 9.6 \times$ $10^{-6}$, and $1.23 \times 10^{-5}$ at all weather conditions respectively. At the third VLC hop, the BER of $1.03 \times 10^{-5}, 1.41 \times 10^{-5}$, and $1.17 \times 10^{-5}$ are competitive to RF communication BER at all weather conditions except for the fog. While at higher VLC hops, the BER gets higher than RF.

Figure 7 illustrates the system overall throughput due to the VLC percentage in each simulation versus the RF percentage at different weather conditions. As in equation (5), we add or remove the transfer function $H$ of each weather condition to study its effect. In clear weather (i.e., only the line- 
of-sight transfer function is considered), the pure VLC channel communication data rate of $355 \mathrm{Mbit} / \mathrm{s}$ is about three times that of pure RF channel communication of $108 \mathrm{Mbit} / \mathrm{s}$.

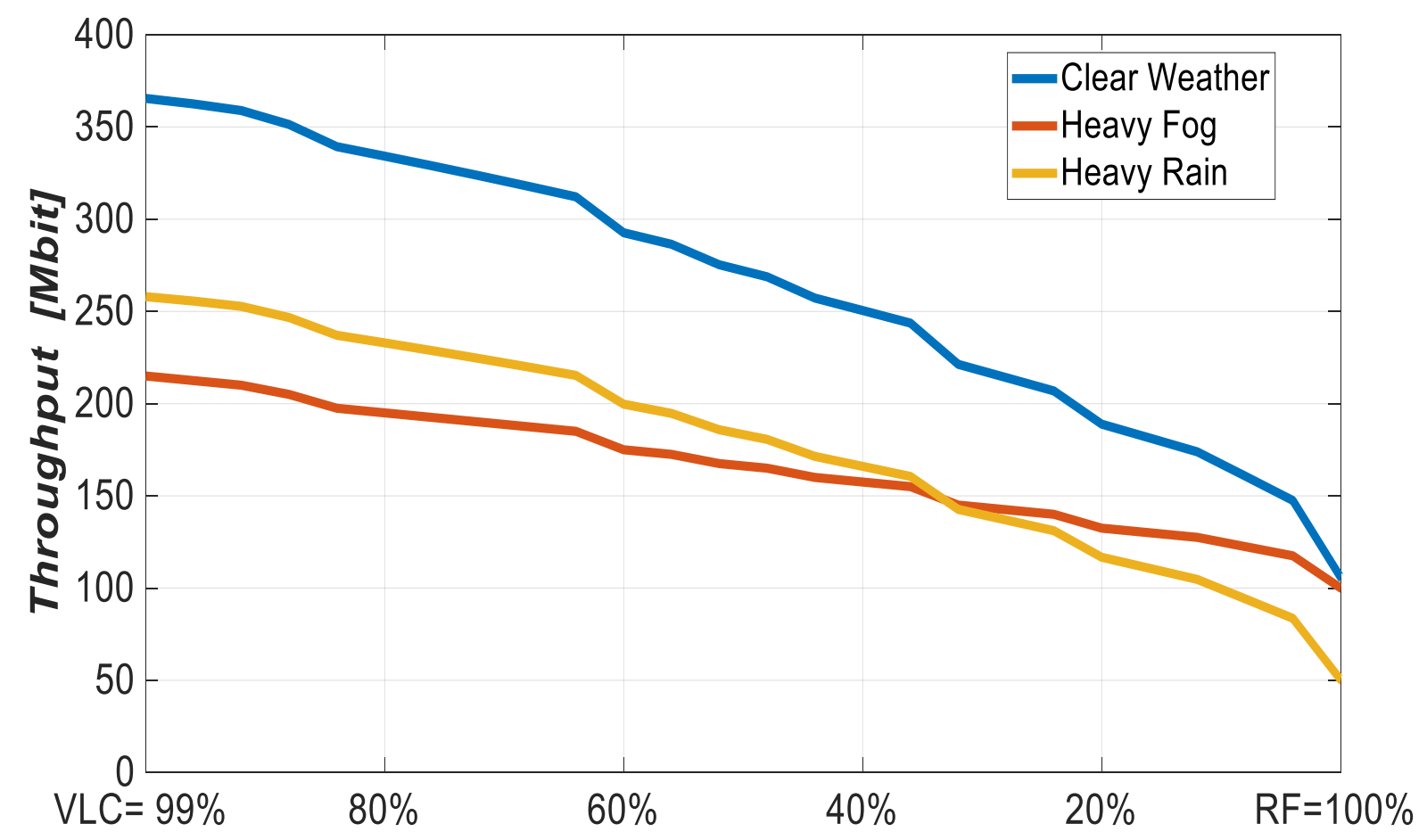

Figure 7 the throughput at the VLC percentage

In heavy fog (i.e., both the line-of-sight transfer function and fog effect transfer function are considered), the pure VLC channel communication data rate of $206 \mathrm{Mbit} / \mathrm{s}$ amounts to about twice that of pure RF communication $98 \mathrm{Mbit} / \mathrm{s}$. In heavy rain (i.e., both the line-of-sight transfer function and rain effect transfer function are considered), the pure VLC channel communication data rate of $257 \mathrm{Mbit} / \mathrm{s}$ is five times that of the pure RF channel communication with $48 \mathrm{Mbit} / \mathrm{s}$. Consequently, increasing the percentage of time the system is working in the RF communication, it suffers from decreased overall throughput at most weather conditions.

Figure 8 illustrates the delay of VLC and RF communication. In both RF and single-hop VLC, a small delay of $4.9 \times 10^{-4} \mathrm{Sec}$ and $4.3 \times 10^{-4} \mathrm{Sec}$ respectively are noticed. In contrast, the multihops delay increases as the number of hops increases because it resamples a multiple of one hope delay. Some critical information needs to be transferred with a very small delay. Then, a higher 
number of hops is not a practical approach for such critical information and it should be covered through RF communication.

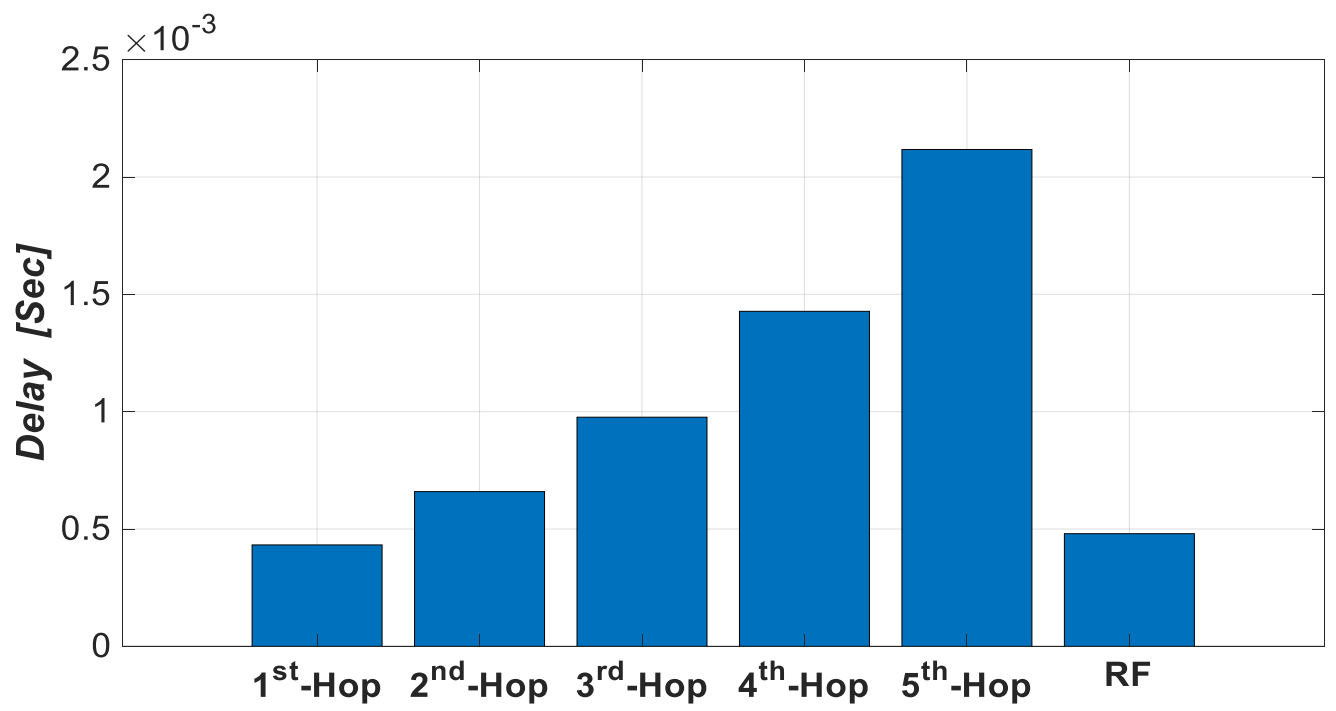

Figure 8 the delay at VLC and RF communication

\section{CONCLUSION}

In this paper, we have introduced the problem facing car-to-car communication and why the RF communication alone could not fulfill the needed requirements within the next decade. Then, we have demonstrated the VLC communication advantages such as higher data rate which can cover modern applications need and the easy setup requirements (i.e., camera and LED). After that, the proposed scheme in this paper combines the advantages of both the VLC communication and the RF communication for car-to-car applications to have a higher data rate, higher range coverage, and smaller BER. Our results showed that VLC can achieve up to four times the RF throughput. Consequently, increasing the percentage of time the system is working in the RF communication condition not only suffers from higher BER but also suffers from decreased overall throughput. However, due to the car-to-car critical communication situations, it is necessary to have an RF communication backup.

\section{Abbreviations:}

Visible Light Communication (VLC), Multi-Input Multi-Output (MIMO), Light Emitting Diode (LED), Bit Error Rate (BER), Radio Frequency (RF), Coalitional Game (CG), Engine Control Unit 
(ECU), Third Generation Mobile Network (3G), Fourth Generation Mobile Network (4G), Fifth Generation Mobile Network (5G), Analytic Hierarchy Process (AHP), decibel (dB), Signal to Noise Ratio (SNR), ON-OFF Key modulation (OOK), Frame Per Second (FPS), Additive White Gaussian Noise (AWGN), Quadrature Amplitude Modulation (QAM), and Rain Rate (RR).

\section{Declarations}

\section{Acknowledgments:}

No additional contributors.

\section{Authors' contributions:}

The author Nermeen M. Okasha presented the paper idea, wrote the paper sections, build the MATLAB model, and discussed the findings. The author Abdel Halem A. Zekry reviewed the paper idea and sections, supervised the model, and proofread the manuscript. The author Fatma A. Newagy reviewed the manuscript. All authors read and approved the final manuscript.

\section{Funding:}

Not applicable.

\section{Availability of data and materials:}

Not applicable.

\section{Ethics approval and consent to participate:}

Not applicable.

\section{Competing interests:}

The authors declare that they have no competing interests.

\section{REFERENCES}

[1] Ucar, Seyhan, et al. "Dimming support for visible light communication in intelligent transportation and traffic system." NOMS 2016-2016 IEEE/IFIP Network Operations and Management Symposium. IEEE, 2016. 
[2] Aini, Qurratul, et al. "Investigation of intelligent transport system with optical vehicleto-vehicle communication." International Research Journal on Advanced Science Hub 2 pp. 132-137, 2020.

[3] Li, Xicong, et al. "A $40 \mathrm{Mb} / \mathrm{s}$ VLC System Reusing an Existing Large LED Panel in an Indoor Office Environment." p.1697, no.5, 2021.

[4] Eldeeb, Hossien B., Sadiq M. Sait, and Murat Uysal. "Visible light communication for connected vehicles: How to achieve the omnidirectional coverage?" IEEE Access 9 (2021): 103885-103905.

[5] Cosovanu, Lucian-Mihai, and Adrian Done. "Development of visible light communication system for automotive applications based on organic light emitting diode panels." In 2020 International Conference on Development and Application Systems (DAS), pp. 84-89. IEEE, 2020.

[6] Georlette, V., Moeyaert, V., Bette, S., \& Point, N. (2020, July). Visible light communication challenges in the frame of smart cities. In 2020 22nd International Conference on Transparent Optical Networks (ICTON) (pp. 1-4), 2020.

[7] K. Lennart, and M. Zuniga, "Shine: A step towards distributed multi-hop visible light communication," In 2015 IEEE $12^{\text {th }}$ International Conference on Mobile Ad Hoc and Sensor Systems, pp. 235-243, IEEE, 2015.

[8] K. Wook, and S. Jung, "Vehicle positioning scheme using V2V and V2I visible light communications," In 2016 IEEE 83rd Vehicular Technology Conference (VTC Spring), pp. 1-5, IEEE, 2016.

[9] B. Rebecca, C. Geldard, N. Hassan, A. Burton, and P. Luo, "Comparison of LED illumination patterns for camera based car to car communications," International Symposium on Communication Systems, Networks and Digital Signal Processing (CSNDSP), pp. 1-6, April 2016.

[10] P. Ioan, A. Lavric, and E. Coca, "VLC for Vehicular Communications: A Multiple Input Multiple Output (MIMO) Approach,” In 2018 International Conference on Development and Application Systems (DAS), pp. 134-137, IEEE, 2018. 
[11] F. Tian, S. Zhu, Y. Chen, and Xuzhen Zhu, "Traffic-Aware Relay Vehicle Selection in Millimeter-Wave Vehicle-to-Vehicle Communication," IEEE Wireless Communications Letters 8, pp. 400-403, 2018.

[12] E. Elizabeth, A. Burton, N. Hassan, M. Abadi, Z. Ghassemlooy, and S. Zvanovec, "Experimental Investigation of the Effects of Fog on Optical Camera-based VLC for a Vehicular Environment," In 2019 15th International Conference on Telecommunications (ConTEL), pp. 1-5, IEEE, 2019.

[13] Zadobrischi, Eduard. "System Prototype Proposed for Vehicle Communications Based on VLC-RF Technologies Adaptable on Infrastructure." 2020 International Conference on Development and Application Systems (DAS). IEEE, 2020.

[14] Shen, Xiaotong, and Harald Haas. "Vehicle to Infrastructure VLC Channel Models." 2020 IEEE 91st Vehicular Technology Conference (VTC2020-Spring). IEEE, 2020.

[15] Ardi, Arnez Pramesti, et al. "VLC-Based Car-to-Car Communication." Jurnal Elektronika dan Telekomunikasi pp.6-22, 2020.

\section{Figure Title and Legend}

Figure 1: The proposed framework of 80 cars connects through VLC/RF within 6 lanes road. car-to-car communication system comprises a framework of 80 cars within six lanes road with three lanes in one direction and the other three lanes in the opposite direction. green arrows represent single-hop VLC, red arrows represent multi-hop VLC, yellow arrows represent multi-hop VLC to overcome the field of view problem while yellow lightening represents single-hop RF. The selected road distance is $25 \mathrm{~km}$. Each car has a variable speed from $60 \mathrm{~km}$ to $100 \mathrm{~km}$.

Figure 2: The VLC Channel Characteristics at different weather conditions.

The received signal is affected by the channel attenuation coefficients such as fog, rain, dust, and snow. Moreover, the additive white Gaussian noise from light sources such as the sun, commercial boards, and Lampposts.

Figure 3: The proposed scheme flowchart. 
The proposed flow chart builds a framework of the road and randomly allocates 80 cars within the road. Then, set a random speed for each car and choose one car and sender and another as the receiver. The generated data in the sender car tries to communicate with the receiver through VLC single hop. Then, the car increases the number of hops if it fails to close the connection. However, in some weather conditions, the VLC communication cannot succeed. Then, if the maximum number of hops is reached, the scheme switches the communication type to RF.

\section{Figure 4: Multi-hop car-to-car communication.}

Cars VLC communication is either through headlights or tail lights. Then, no communication is possible between neighboring cars. Consequently, the transmitter source (i.e., white car) communicates through the repeater (i.e., blue car) to reach the receiver (i.e., yellow car). Therefore, multi-hop communication forms a chain of repeaters between the source and destination.

\section{Figure 5: The BER versus distance}

In VLC communication, increasing the distance leads to higher BER until approximately $100 \mathrm{~m}$ (i.e., the maximum allowed distance for VLC communication) in each hop. In VLC first hop, a small BER is noticed, in the second hop, the error increased due to the repeater amplify and forward scheme errors while at higher hops have higher max errors. In contrast, RF communication has near-constant BER at distances from $100 \mathrm{~m}$ (i.e., after the VLC hop maximum communication distance) up to 200 m. we consider no communication at distances greater than $200 \mathrm{~m}$ to ensure the minimized interference in RF (omnidirectional radiation pattern) and to maximize the frequency reuse in car-tocar RF communication.

\section{Figure 6: The BER at different weather conditions}

The BER at different weather conditions (i.e., clear weather, heavy fog, and heavy rain) in all communication scenarios. In general, the VLC and RF communication at clear weather conditions have the lowest BER compared to other weather conditions. While, the VLC communication at foggy weather has the highest BER at VLC with low noticeable effect on RF because the fog even if 
it minimizes the visibility in the visible light band, does not block the other electromagnetic radiations such as RF communication.

\section{Figure 7: The throughput at the VLC percentage}

the system overall throughput due to the VLC percentage in each simulation versus the RF percentage at different weather conditions. As in equation (5), we add or remove the transfer function of each weather condition to study its effect. In clear weather (i.e., only the line-of-sight transfer function is considered), the pure VLC channel communication data rate is about three times that of the pure RF channel communication data rate.

\section{Figure 8: The delay at VLC and RF communication}

This figure illustrates the delay of VLC and RF communication. In both RF and single-hop VLC a small delay is noticed. In contrast, the multi-hops delay increases as the number of hops increases because it resamples a multiple of one hope delay. Some critical information needs to be transferred with a very small delay. Then, a higher number of hops is not a practical approach for such critical information and it should be covered through RF communication. 